\title{
A Stochastic Optimization Model for the Invest- ment of Savings Account Deposits
}

\author{
Bruce Forrest, ${ }^{1}$ Karl Frauendorfer ${ }^{2}$ and Michael Schürle ${ }^{2}$ \\ ${ }^{1}$ Swiss Bank Corporation, Corporate Financial Management, Zurich, Switzerland \\ ${ }^{2}$ University of St. Gallen, Institute of Operations Research, St. Gallen, Switzerland
}

\section{Introduction}

In a bank's balance sheet, non-maturing accounts can be characterized as follows: (1) There is no contractual maturity on this kind of account, allowing customers to withdraw or repay their investments or credits at any point in time at no penalty. (2) The customer rate is not indexed to certain interest rates or prices of traded instruments but adjustable to market conditions as a matter of policy. The most common examples include some forms of savings accounts or non-fixed mortgages as they are widespread in Europe and the U.S. These assets and liabilities are not only sensitive to changes in interest rates but have also embedded call or put options that may be excercised by the customer, making their management a particularly ambitious task. A homeowner, e.g., has the option to prepay the outstanding balance of his mortgage and hence, call the security.

In practise, customer behavior depends strongly on the current market environment. In case of variable rate mortgages, the total volume that has to be refinanced by the bank is positively correlated with interest rates. When the latter are low, there is a sharp drop in demand since customers switch to fixed rate mortgages in order to hedge against a future rise. On the contrary, the volume of savings deposits increases since their yields are relatively attractive compared to short-term securities. Therefore, even institutional investors like pension funds prefer these deposits instead of direct investments in money market instruments. In such a situation, it is difficult for the financial management to find a combination of fixed-income instruments that provides a sufficient margin and takes into account the risk that a significant share of the deposits is withdrawn.

It is often argued that a bank's balance sheet may be viewed as a long position (assets) and a short position (liabilities) in bonds of different maturities. Duration matching is a well known technique to reduce the interest rate risk one is exposed to. In the classical sense, this approach hedges parallel shifts of the yield curve, assuming that interest rates are driven by a single source of uncertainty - the level factor. Many authors have shown that multi-factor models are better suited to describe the behavior of interest rates. E.g., Litterman/Scheinkman [7] identified 
two additional factors that also explain tilt and humped movements of the yield curve. Various extensions of the duration concept like the key rate durations due to Ho [4] have been introduced in order to capture more complex interest rate dynamics. However, duration based approaches require permanent rebalancements of portfolios to immunize the positions, in particular if implicit options are embedded. Increasing transaction costs are the consequence.

In the case of non-maturing accounts, it is also difficult to find adequate duration measures due to uncertain customer behavior. These shortcomings have motivated the replicating portfolio approach (see [8]). Instead of identifying the various sources of risk, one tries to mimic the behavior of the target account in order to capture its characteristics. This is achieved by finding a portfolio of fixed income securities whose return replicates the customer rate of the relevant asset or liability position. Transaction costs remain low if only liquid standard instruments like money market and swap positions are used. These are held until maturity to avoid rebalancing transactions. Maturing funds are renewed at the same maturity. Prepayment and withdrawal risks are implicitly taken into account as the volume of the replicating portfolio has to coincide with the volume of the target account at all points in time. The weights are determined through minimizing the tracking error over a historic period. This approach transforms uncertain cash flows into apparently certain ones, allowing the bank to manage them like normal maturing accounts.

The latter approach is currently used at SBC to manage their non-maturing accounts positions. The advantageous features are low transaction costs, easy implementability and transparency. However, the competition and the permanent invention of complex financial products have increased the need for new concepts within asset \& liability management (ALM). Stochastic optimization models take into account the correlation between interest rates and volume. This helps increase the bank's margin and, simultaneously, manages the various risks properly since it allows for active response to changes in the market environment. For some successful applications of stochastic programming within ALM and financial planning, it is referred to [1], [5], [6], [9] and [10]. In this paper, a multistage model for the management of savings account deposits is introduced. The results presented in section 3 refer to a case study based on the interest rate evolution over 7 years.

\section{Optimization Model}

The formulation of the optimization model is straightforward: $\mathcal{D}=\{1,2, \ldots, D\}$ denotes a set of maturity dates for the fixed-income securities held in the portfolio. Investment opportunities are given by a set of some standard maturities $\mathcal{D}^{S} \subset \mathcal{D}$ that are traded in the market. The amount of a long position with maturity $d \in \mathcal{D}^{S}$ raised at $t$ is denoted $v_{t}^{d,+}$, a short position is given by $v_{t}^{d,-}$. The objective is to maximize the income from long and short positions. Let the sum of discounted interest payments per unit of investments and borrowings of maturity $d$ within the planning horizon 
be denoted by $\varphi_{t}^{d,+}$ and $\varphi_{t}^{d,-}$. The latter are determined by the realizations of a $K$-dimensional process $\left(\eta_{t} ; t \in[0, T]\right)$ which drives the stochastic evolution of the yield curve. Here, $K=3$ key rates are modelled as correlated Brownian motions in discrete time. The remaining rates are interpolated, allowing the model to reflect shift, tilt, and humped movements of the yield curve. The functional relationship between $\eta_{t}$ and $\varphi_{t}^{d,+}, \varphi_{t}^{d,-}$ incorporates the sensitivity of interest rates subject to changes in the risk factors, transaction costs, a bid-ask spread and the discount mechanism. The volume change $\left(\xi_{t} ; t \in[0, T]\right)$ is normally distributed and correlated with the key rates for mapping customer behavior. $P$ is the joint probability measure of $(\eta, \xi)$ associated with time $t=1, \ldots, T$, i.e., $\eta=\left(\eta_{1}, \ldots, \eta_{T}\right), \xi=\left(\xi_{1}, \ldots, \xi_{T}\right)$. The corresponding stochastic multistage program reads as

$$
\begin{aligned}
& \max \int \sum_{t=0}^{T} \sum_{d \in \mathcal{D}^{S}}\left(\varphi_{t}^{d,+} \cdot v_{t}^{d,+}-\varphi_{t}^{d,-} \cdot v_{t}^{d,-}\right) d P(\eta, \xi) \\
& \text { s.t. (1a) } v_{t}^{d}-v_{t-1}^{d+1}-v_{t}^{d,+}+v_{t}^{d,-}=0 \\
& \text { (1b) } v_{t}^{d}-v_{t-1}^{d+1} \quad=0 \\
& \text { (2) } x_{t}-\sum_{d \in \mathcal{D}} v_{t}^{d} \quad=0 \\
& \text { (3) } x_{t}-x_{t-1}=\xi_{t} \\
& \text { (4) } \quad \sum_{d \in \mathcal{D}^{S}} v_{t}^{d,+}-\sum_{d=1}^{M} v_{t-1}^{d} \leq \xi_{t} \\
& \text { (5a) } \quad 0 \leq v_{t}^{d,+} \leq \ell_{t}^{d,+} \\
& \text { (5b) } \quad 0 \leq v_{t}^{d,-} \leq \ell_{t}^{d,-} \\
& \text { (6a) } \quad v_{t}^{d,+}, v_{t}^{d,-} \quad \text { are nonanticipative } \\
& \text { (6b) } \quad x_{t}, v_{t}^{d} \in \mathbb{R} \text { are nonanticipative } \\
& \begin{aligned}
t & =0,1, \ldots, T ; \forall d \in \mathcal{D}^{S} \\
t & =0,1, \ldots, T ; \forall d \notin \mathcal{D}^{S} \\
t & =0,1, \ldots, T \\
t & =1,2, \ldots, T \\
t & =0,1, \ldots, T \\
t & =0,1, \ldots, T ; \forall d \in \mathcal{D}^{S} \\
t & =0,1, \ldots, T ; \forall d \in \mathcal{D}^{S} \\
t & =0,1, \ldots, T ; \forall d \in \mathcal{D}^{S} \\
t & =0,1, \ldots, T ; \forall d \in \mathcal{D} .
\end{aligned}
\end{aligned}
$$

The sum of long and short positions $v_{t}^{d}$ at $t$ maturing after $d$ periods is determined by (1a) and (1b). $v_{-1}^{d}$ denotes the initial portfolio composition from decisions in the past. Constraint (2) requires that the total savings volume $x_{t}$ equals the sum of long and short positions $v_{t}^{d}$ for any $t$. Equation (3) characterizes the stochastic customer behavior. Liabilities may be raised for financing additional investments at an amount equal to the sum of funds maturing at $t+1, \ldots, t+M-1$. These short sales may be restricted by a positive integer $M$ in (4) ( $M=1$ prohibits short sales). Liquidity restrictions are taken into account by imposing upper limits $\ell_{t}^{d,+}, \ell_{t}^{d,-}$ for investments and borrowings in (5a) and (5b). Finally, the nonanticipativity constraints (6a) and (6b) require that two scenarios which share the same history up to time $t$ must have identical decisions and state variables up to $t$.

It can easily be seen from the problem formulation that the risk factors $\eta_{t}$ appear only in the objective function while the volume change $\xi_{t}$ affects solely the righthand side of the constraints. In particular, the coefficients on the left side are deterministic. Together with the assumptions on the probability distribution of the risk factors, this ensures that the value functions at $t$ are convex-concave saddle functions in $\left(\eta_{t}, \xi_{t}\right)$ (for details, see [2]). The underlying saddle structure motivates the application of barycentric approximation, which approximates the stochastic evolution of interest rates and volume change by two scenario trees providing upper 
and lower bounds for the original problem. Setting $\omega_{t}=\left(\eta_{t}, \xi_{t}\right)$, each of whose scenario trees and the associated path probabilities are characterized as

$$
\begin{aligned}
\mathcal{A} & =\left\{\omega^{T} \in \Omega \mid \omega_{t} \in \mathcal{A}_{t}\left(\omega^{t-1}\right) \forall t>1\right\} \\
q\left(\omega^{T}\right) & =\prod_{t=1}^{T} q_{t}\left(\omega_{t} \mid \omega^{t-1}\right),
\end{aligned}
$$

where $\mathcal{A}_{t}\left(\omega^{t-1}\right)$ denotes the set of finitely many outcomes for $\omega_{t}$ conditioned on $\omega^{t-1}$. $Q$ is the joint probability measure of $\mathcal{A}$, and $Q_{t}\left(\cdot \mid \omega^{t-1}\right)$ the associated conditional discrete probability measure.

The characteristics of the approximation of the (conditional) probability measure $P_{t}\left(\cdot \mid \omega^{t-1}\right)$ are conceptually outlined as follows: The support of $\left(\omega_{t} \mid \omega^{t-1}\right)$ is covered by the Cartesian product $\Omega_{t}=\Theta_{t} \times \Xi_{t}$ of simplices, socalled $\times$-simplices. $\Theta_{t} \in \mathbb{R}^{K}$ and $\Xi_{t} \in \mathbb{R}^{L}$ are assumed to be regular simplices whose vertices are denoted $a_{\nu}, \nu=$ $0,1, \ldots, K$, and $b_{\mu}, \mu=0,1, \ldots, L$. The probability measure $P_{t}$ on $\Omega_{t}$ induces mass distributions $\mathcal{M}_{\nu}$ with associated generalized barycenters $\xi_{\nu}$ on the $L$-dimensional simplices $a_{\nu} \times \Xi_{t}$. As for $\nu=0,1, \ldots, K$ the mass distributions $\mathcal{M}_{\nu}$ add up to a probability distribution, a discrete probability measure $Q_{t}^{u}$ on $\Theta_{t} \times \Xi_{t}$ is derived when probability $\mathcal{M}_{\nu}\left(\left\{a_{\nu}\right\} \times \Xi_{t}\right)$ is assigned to point $\left(a_{\nu}, \xi_{\nu}\right)$. Substituting $Q_{t}^{u}$ in the original stochastic program yields an upper bound for the expectation. Analogously, $P_{t}$ induces mass distributions $\mathcal{M}_{\mu}$ with associated generalized barycenters $\eta_{\mu}$ on the $K$-dimensional simplices $\Theta_{t} \times\left\{b_{\mu}\right\}$. Again, the mass distributions $\mathcal{M}_{\mu}$ add up to a probability distribution for $\mu=0,1, \ldots, L$, yielding a discrete probability measure $Q_{t}^{l}$ on $\Theta_{t} \times \Xi_{t}$ by assigning probability $\mathcal{M}_{\mu}\left(\Theta_{t} \times\left\{b_{\mu}\right\}\right)$ to point $\left(\eta_{\mu}, b_{\mu}\right)$. Substituting $Q_{t}^{l}$ for $P_{t}$ yields a lower bound for the expectations (for details, see [3]).

Both approximate problems are treated as deterministic multistage programs. This makes a broad class of stochastic programming algorithms available which allow the block structure and associated sparsity to be exploited. The total numbers of scenarios within the scenario trees is given by $[(L+1) \cdot h]^{T}$ for the lower approximation and by $[(K+1) \cdot h]^{T}$ for the upper approximation, where $h>0$ is the number of $\times$ simplices used at $t=1, \ldots, T$ to cover the support of $\left(\omega_{t} \mid \omega^{t-1}\right)$. Clearly, the goodness of discretization increases with $h$. However, the problem size grows exponentially with $h$ and the planning horizon $T$.

\section{Case Study}

In order to assess the performance of the stochastic optimization model and to compare it with the replicating portfolio approach, a case study was set up based on historic monthly interest data of a 7 year horizon (see Figure 1 ). At $t=0$, the volume to be invested was set to 30 billion with a given maturity structure. The volume change was characterized by the behavior of the savings account position. Investment opportunities consisted of interest rate swaps with maturities of $1,2,3$, 4, 5, 7, and 10 years. Taking liquidity restrictions into account, upper limits were set to 500 mio. for swaps up to 5 years and to 200 mio. for 7 and 10 years. 

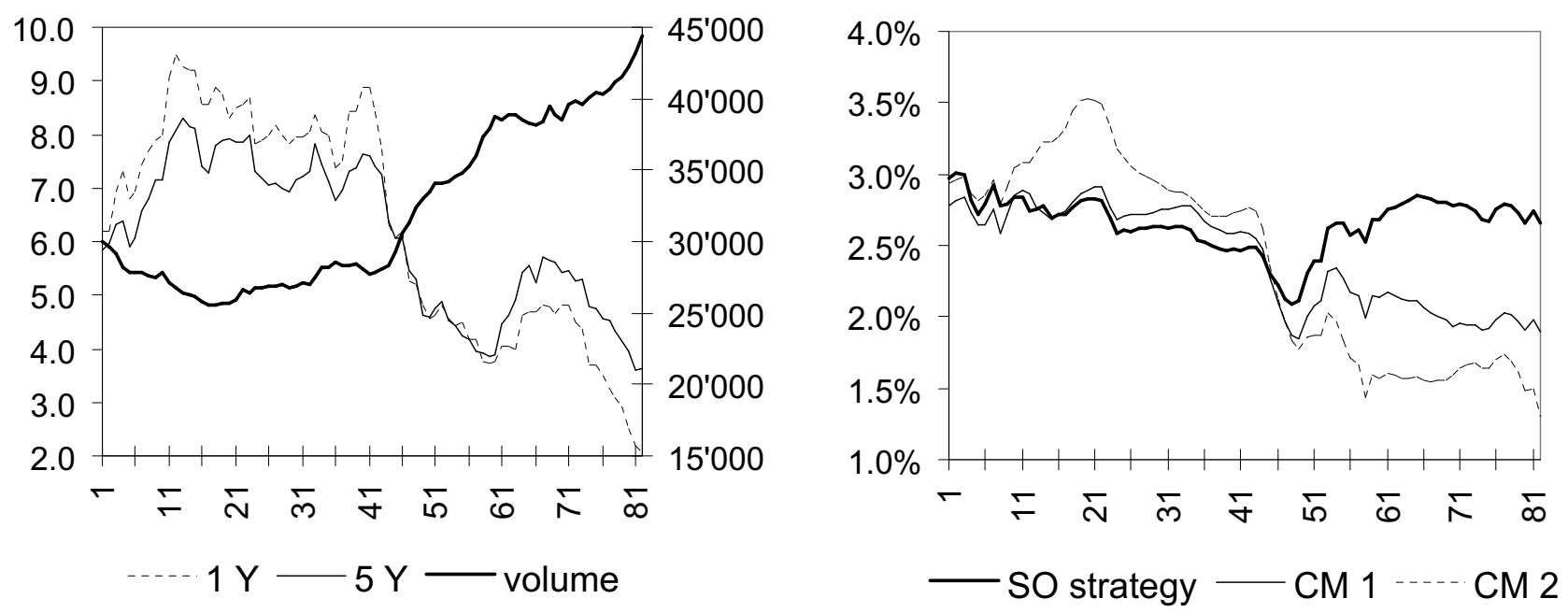

Figure 1: Evolution of interest rates, volume in mio. (left), and margin (right).

The optimization was based on a set of 11 stress scenarios characterized by drifts of the underlying multidimensional stochastic process for the three key rates, reflecting shift and tilt movements of the yield curve. In general, this results in several optimal solutions conditioned on the different assumptions on key rate drifts. Risk analysis is employed for valuing the decisions at $t=0$ and their impact on future periods. This reveals a profit \& loss pattern w.r.t. the 11 stress scenarios and helps assess dominant policies for $t=0$, taking into account the risk of nonanticipated shift and tilt movements. The performance of the derived dynamic policy (SO strategy) compared with two static portfolios CM 1 \& CM 2 determined by means of the replicating portfolio approach is shown in Figure 1 and the following table:

\begin{tabular}{c|c|c} 
Policy & avg. margin [\%] & std. dev. [\%] \\
\hline SO strategy & 2.659 & 0.188 \\
CM 1 & 2.414 & 0.358 \\
CM 2 & 2.399 & 0.696
\end{tabular}

The first constant mix consists of maturities of 2 and 5 years $(50 \%, 50 \%)$, the second of 1,2 , and 5 years $(35 \%, 35 \%, 30 \%)$. The stochastic optimization model clearly outperforms both constant mixes since the margin is increased by approximately 25 BP. In addition, the margin's standard deviation is reduced significantly compared to the static portfolios. It is noted that volatility is not considered in the model's objective but incorporated implicitly by the risk analysis mentioned above.

\section{Conclusions}

The performance of the stochastic optimization model presented in this paper encouraged SBC to apply it for the management of their savings accounts as well as 
for refinancing their non-fixed (Swiss style) mortgages. Results of the case study indicate that a dynamic policy is superior to a static one. In particular, by exploiting the correlations between interest rates and uncertain cash flows, various sources of uncertainty inherent to non-maturing accounts like prepayment or withdrawal risk may be taken into account in dynamic portfolio strategies. Therefore, the stochastic optimization approach is well suited for a broad class of investment problems being characterized by (cross and/or serial) correlations of risk factors, e.g., cash management in insurance companies where premium payments exhibit seasonal behavior. Other types of risk (credit, currency, etc.) may also be considered if appropriate.

The savings account model may be seen as a first step towards a general asset \& liability management model comprising a bank's complete balance sheet and optimizing the investment and refinancing decisions with respect to interest rate risk exposure. Additional constraints may be imposed to limit the absolute risk exposure of certain positions in order to comply with regulatory restrictions concerning capital requirements. The gained experiences allow one to conclude that multistage stochastic programming helps overcome many difficulties of modeling dynamic decision making within asset \& liability management under uncertainty. It may be seen as a supplementary tool to modern approaches of corporate finance.

\section{References}

[1] D.R. Cariño, T. Kent, D.H. Myers, C. Stacy, M. Sylvanus, A.L. Turner, K. Watanabe, and W.T. Ziemba. The Russell-Yasuda Kasai Model: An Asset/Liability Model for a Japanese Insurance Company Using Multistage Stochastic Programming. Interfaces, 24:29-49, 1994.

[2] K. Frauendorfer. Stochastic Two-Stage Programming. Springer, 1992.

[3] K. Frauendorfer. Barycentric Scenario Trees in Convex Multistage Stochastic Programming. Mathematical Programming (Ser. B), 75(2):277-293, 1996.

[4] T. Ho. Key Rate Durations: Measures of Interest Rate Risks. Journal of Fixed Income, 2:29-44, 1992.

[5] J.G. Kallberg, R.W. White, and W.T. Ziemba. Short Term Financial Planning under Uncertainty. Management Science, 28(6):670-682, 1982.

[6] M.I. Kusy and W.T. Ziemba. A Bank Asset and Liability Management Model. Operations Research, 34:356-376, 1986.

[7] R. Litterman and J. Scheinkman. Common Factors Affecting Bond Returns. Journal of Fixed Income, 1:54-61, June 1991.

[8] M. Spillmann. Risk-Return-Philosophie im Asset and Liability Management. Invest, 78-79, November 1996.

[9] S.A. Zenios and W.T. Ziemba. Financial modeling. Management Science, 38, 1992.

[10] W.T. Ziemba and J.M. Mulvey (eds.). Worldwide Asset and Liability Management. Cambridge University Press, 1997. 